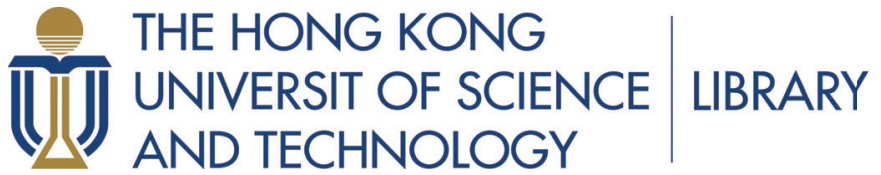

\section{HKUST SPD - INSTITUTIONAL REPOSITORY}

Title Enabling room-temperature solid-state lithium-metal batteries with fluoroethylene carbonate-modified plastic crystal interlayers

Authors Lu, Ziheng; Yu, Jing; Wu, Junxiong; Effat, Mohammed Badrt Mostafa Kamel; Kwok, Chin To Stephen; Lyu, Yuqi; Yuen, Ming Fai; Ciucci, Francesco

Source Energy Storage Materials, v. 18, March 2019, p. 311-319

Version Accepted Version

DOI $\quad$ 10.1016/j.ensm.2018.08.021

Publisher Elsevier

Copyright @ 2018 Elsevier

License CC BY-NC-ND

This versionis availableat HKUST SPD - Institutional Repository (https://repository.ust.hk/ir)

If it is the author's pre-published version, changes introduced as a result of publishing processes such as copy-editing and formatting may not be reflected in this document. For a definitive version of this work, please refer to the published version. 


\title{
Enabling room-temperature solid-state lithium-metal batteries with fluoroethylene carbonate-modified plastic crystal interlayers
}

\begin{abstract}
Solid-state batteries (SSBs) with $\mathrm{Li}_{7} \mathrm{La}_{3} \mathrm{Zr}_{2} \mathrm{O}_{12}$ (LLZO) ceramic oxide electrolytes are attracting significant interest because of LLZO's non-flammability, excellent ionic conductivity, relative electrochemical stability against Li metal anodes, and processability in air. However, the poor solid-solid contact between the electrolyte and the electrodes leads to large interfacial impedances, which are detrimental to the functioning of LLZO-based SSBs. In this work, we modified the electrode|Ta-doped-LLZO (LLZTO) interfaces by employing a plastic crystal interlayer based on succinonitrile with a fluoroethylene carbonate additive. The interlayer, which can be easily applied, drastically reduces the interfacial resistances and allows the stable operation of Li-metal based batteries. A $\mathrm{Li}|\mathrm{LLZTO}| \mathrm{LiFePO}_{4}$ battery with this interlayer can stably cycle at room-temperature for 50 times at $0.1 \mathrm{C}$ while still retaining a capacity of 140 $\mathrm{mAh} \mathrm{g}^{-1}$. The symmetric Li|LLZTO|Li cell with the interlayer can cycle at $0.2 \mathrm{~mA} \mathrm{~cm}^{-2}$ for over 150 hours. It also has a higher critical current density for the growth of dendrites compared with an analogous cell without the interlayer. In short, this work provides a facile and efficient methodology to enhance the effective $\mathrm{Li}$ transportation rates at the electrode/electrolyte interfaces of SSBs and can be readily applied to other types of electrolytes beyond LLZO.
\end{abstract}

\section{Introduction}

Li-ion batteries (LIBs) are widely used as energy storage media because of their high energy density, high power density, and slow self-discharge rates. [1, 2] In fact, they have been dominating the market of portable electronics since their launch by Sony in the 1990s. [2] LIBs have also emerged as the technology of choice for electric vehicles. [3, 4] The fast-growing market for LIBs, especially for high power applications, poses serious safety concerns because the aprotic electrolytes used commercially are extremely volatile, toxic, and flammable, 
potentially leading to fires and explosions when managed improperly. [3] Ceramic solid-state electrolytes (SSEs) are considered as a promising solution to these challenges because they are non-flammable and have the potential to be paired with Li metal anodes and high-voltage cathodes to achieve high energy densities. [5]

To date, two major bottlenecks still hinder the practical use of ceramic-based SSBs, namely, 1) the low ionic conductivity of SSEs; and 2) the large interfacial resistance between electrodes and the SSEs. The former bottleneck requires the development of highly ionic conductive materials. [6, 7] Over the past decade, many SSEs with good ionic conductivity have been discovered, including $\mathrm{Li}_{10} \mathrm{GeP}_{2} \mathrm{~S}_{12}$ (LGPS), [8, 9] $\mathrm{Li}_{7} \mathrm{La}_{3} \mathrm{Zr}_{2} \mathrm{O}_{12}$ (LLZO) [10, 11], $\mathrm{Li}_{x} \mathrm{La}_{2 / 3-\mathrm{x} / 3} \mathrm{TiO}_{3}$ (LLTO) $[12,13]$, and $\mathrm{Li}_{2} \mathrm{O}-\mathrm{Al}_{2} \mathrm{O}_{3}-\mathrm{TiO}_{2} / \mathrm{GeO}_{2}-\mathrm{P}_{2} \mathrm{O}_{5}$ (LATP/LAGP) [14, 15$]$. Some of these materials even have room temperature (RT) conductivities that surpass those of conventional liquid electrolytes. For example, LGPS has an ionic conductivity of $10^{-2} \mathrm{~S} \mathrm{~cm}^{-1}$ at RT. [9] For highly conductive SSEs, the major challenge is the large interfacial resistance between SSEs and electrodes originating from the poor solid-solid contacts and the electrochemical incompatibility between the electrolyte and electrodes. [16] Shen and coworkers found that rigid interfacial contacts in SSBs result in large interfacial resistances and deterioration of battery performance. [17] This issue is especially severe on the cathode side because the active material undergoes significant volumetric changes during operation. [18] Such cycled expansions and contractions deteriorate the contacts and, in turn, lead to an irreversible capacity loss as evidenced by a recent study from the Janek group. [19] Besides the poor contact, the electrochemical incompatibility of electrodes and SSEs may also have a detrimental impact on the SSB. $[16,20,21]$ Yasutoshi et al. found that the contact between $\mathrm{LiCoO}_{2}$ and LLZO triggers the $\mathrm{Co}$ and La inter-diffusion, which, in turn, results in the formation of Li-insulating phases. [22] On the other hand, LLTO and LGPS are known to be unstable with Li. The reaction products are electron conductive, thereby resulting in the progressive degradation of the electrolytes. [21, 23] 
Various methods have been developed to solve these interfacial challenges. To improve the solid-solid contact, one can use ionic conductive materials to fill the gaps between the electrodes and the SSEs. For example, Shingo et al. dispersed $\mathrm{Li}_{3} \mathrm{BO}_{3}$, a low melting-point ionic conductor, at the boundary between LLZO and the $\mathrm{LiCoO}_{2}$ cathode. [24] $\mathrm{Li}$ and co-workers used a soft polymer material at the $\mathrm{Li} \mid \mathrm{Li}_{6.5} \mathrm{La}_{3} \mathrm{Zr}_{1.5} \mathrm{Ta}_{0.5} \mathrm{O}_{12}$ (LLZTO) interface to reduce the interfacial impedance. [25] The Hu and Washman groups found that LLZO is lithiophobic and, consequently, coated the LLZO surface with $\mathrm{Si}, \mathrm{Ge}, \mathrm{Al}, \mathrm{ZnO}$, and $\mathrm{Al}_{2} \mathrm{O}_{3}$ to improve the cycling stability. [26-29] Gao et al. used a plastic crystal material interphase to achieve good contact between the cathode and the electrolyte in a Na-based SSB. [30] Such a battery, composed of a $\mathrm{Na}_{3} \mathrm{Zr}_{2}\left(\mathrm{Si}_{2} \mathrm{PO}_{4}\right)$ electrolyte, a $\mathrm{Na}_{3} \mathrm{~V}_{2}\left(\mathrm{PO}_{4}\right)_{3}$ cathode, and a $\mathrm{Na}$ metal anode, can be cycled at $70{ }^{\circ} \mathrm{C} .[30]$

In this work, we developed a novel plastic crystal interlayer based on the lithium bis(trifluoromethanesulfonyl)imide (LiTFSI) doped-succinonitrile (SN) with fluoroethylene carbonate (FEC) as additives. The interlayer can be easily integrated at both the cathodes $\mid \mathrm{SSE}$ and the anode|SSE interfaces and can efficiently reduce the interfacial resistance of the SSB. The interfacial area-specific resistance (ASR) of a $\mathrm{Li}|\mathrm{LLZTO}| \mathrm{LiFePO}_{4}$ battery dropped from $\sim 13,000 \Omega \mathrm{cm}^{2}$ to $\sim 560 \Omega \mathrm{cm}^{-2}$ after the incorporation of the interlayer. The $\mathrm{Li}|\mathrm{LLZTO}| \mathrm{LiFePO}_{4}$ battery with the interlayer can be cycled at RT for over 50 cycles while maintaining a capacity of $140 \mathrm{mAh} \mathrm{g}^{-1}$. The addition of FEC into the plastic crystal favors its electrochemical stability with Li metal and lowers the critical current density needed for the growth of dendrites in LLZTO. The Li symmetric cell with the interlayer can be cycled at $0.2 \mathrm{~mA} \mathrm{~cm}^{-2}$ for 160 hours without degradation. Overall, this work puts forward a simple strategy to mitigate the interfacial challenges of SSBs by introducing at the electrode/electrolyte interphase a conductive solidstate plastic crystal phase that enables better contact and utilization of the electrodes.

\section{Results and discussion}


The plastic crystal materials were fabricated by first mixing LiTFSI doped-SN (denoted as doped-SN) with FEC additive. We first heated and then mixed three ingredients, i.e., SN, 4 mol.\% LiTFSI salt, and the FEC additive (either 0vol.\%, 2.5vol.\%, 5vol.\%, 10vol.\%, 20vol.\%).

Figure 1 depicts the methodology used to fabricate the plastic crystal materials and the configuration of the SSBs. At $70{ }^{\circ} \mathrm{C}$, the mixtures are in a homogenous, transparent and colorless liquid state (See Figure S1). When the temperature drops to $23{ }^{\circ} \mathrm{C}$ (also referred to RT later), the mixtures with $\leq 5$ vol. $\%$ of FEC solidify. Figure 2 (a) shows the blends at RT, where the hardened samples are non-flowable and translucent. In order to keep the interlayer material solid and, simultaneously, maximize the FEC concentration, we opted to use 5 vol.\% FEC. The mixture of the LiTFSI doped-SN with FEC is denoted as doped-SN+FEC. The experimental data resulting from the use of doped-SN+FEC are also compared with those obtained for doped-SN without FEC (the $0 \%$ vol. mixture is denoted as doped-SN). To characterize the structure of doped-SN and doped-SN+FEC, and to confirm their plastic crystal nature, we carried out X-ray diffraction (XRD). The XRD patterns of doped-SN and dopedSN+FEC show only 2 peaks at $19^{\circ}$ and $27^{\circ}$, see Figure 2 (b). Such patterns closely resemble that of the pure SN in its plastic crystal phase. $[31,32]$ We measured the RT ionic conductivity of doped-SN and doped-SN+FEC using electrochemical impedance spectroscopy (EIS). As shown in Figure $2(\mathbf{c})$, a cylindrically shaped (thickness $=1.6 \mathrm{~mm}$ and diameter $=1.3 \mathrm{~cm})$ piece of doped-SN without FEC has a resistance of $120 \Omega$ at RT. The corresponding ionic conductivity $\left(1.01 \mathrm{mS} \mathrm{cm}^{-1}\right)$ is comparable to that reported by Armand et al. [31] The inclusion of 5 vol.\% FEC does not affect the ionic conductivity significantly; adding 5 vol.\% of FEC lowers the conductivity to $0.92 \mathrm{mS} \mathrm{cm}^{-1}$. Further, the material is highly deformable as shown by manual pressing, see Figure S2. It should be noted, however, that both doped-SN and doped$\mathrm{SN}+\mathrm{FEC}$ materials cannot withstand a significant shear force and cannot form a freestanding thin film. These two issues hinder their usage as separators. Nonetheless, the same two 
properties make them an ideal choice as interlayers between the SSE and the electrodes. SN's deformability allows it to accommodate the volume changes of the active material's particles. Furthermore, the good ionic conductivity of doped-SN allows fast Li transport through the interface and across the extension of the electrodes. It should also be noted that the inclusion of such interlayer is not expected to significantly increase the total costs, because 1) the amount of interlayer material added to the battery is small; and 2) the raw materials are relatively inexpensive ( $\mathrm{SN}$ is manufactured at a large scale for the industrial production of polyamides). [33] Another essential feature of the interlayer material is its electrochemical stability. The interlayers need to be stable against both reduction and oxidation. We tested the electrochemical stability of the plastic crystal material using cyclic voltammetry, see Figure 2 (e). An electrochemical window up to $4.5 \mathrm{~V}$ can be achieved for doped-SN+FEC. However, we must stress that doped-SN by itself is not stable at voltages lower than $1.5 \mathrm{~V}$ or higher than $3 \mathrm{~V}$. In fact, pristine $\mathrm{SN}$ is expected to degrade if placed in contact with Li metal. [31] As mentioned above, the deformable plastic crystal serves as an interlayer between the electrodes and the SSEs. In this work, the SSE is an LLZTO ceramic pellet, synthesized using a conventional solid-state reaction method. [34] The thin LLZTO pellet (thickness $=300 \mu \mathrm{m}$, diameter $=12 \mathrm{~mm}$ ) appears translucent, dense, and relatively flat, see Figure $2(\mathbf{d})$. The EIS spectrum of an LLZTO pellet with a thickness of $1 \mathrm{~mm}$ and cross-sectional area of $1.12 \mathrm{~cm}^{2}$ was tested using Au as the blocking electrodes. The result is shown in Figure 2 (c). The RT ionic conductivity, corresponding to the measured $\sim 400 \Omega$ ionic resistance, is approximately $0.22 \mathrm{mS} \mathrm{cm}^{-1}$. From this conductivity, we estimate that the Ohmic resistance of the $300 \mu \mathrm{m}$ thick pellet with the same cross-sectional area reaches $\sim 120 \Omega$. We will use this value later to evaluate the interfacial resistance of full SSBs.

Since the plastic crystal material is a fluid at $\sim 70{ }^{\circ} \mathrm{C}$, it can be directly cast on the electrode sheets in its liquid state. Solidification follows when the sample is brought to RT. In light of these properties, the interlayer can be easily applied. In our experiments, the electrodes were 
small disks with a diameter of $12 \mathrm{~mm}$. Therefore, we directly dropped $5 \mu \mathrm{L}$ of the melted interlayer material on the electrode surface without casting. Figure 3 (a) shows the morphology of the $\mathrm{LiFePO}_{4}$ cathode covered with the plastic crystal. Compared with the untreated sample (Figure 3 (b)), the plastic crystal filled the voids across the electrode, and the $\mathrm{LiFePO}_{4}$ particles appear to be buried in the translucent interlayer. As we will show below, such morphology enhances the interfacial contact and the cycling stability of the SSB.

To demonstrate that the interlayer decreases the interfacial resistance in an SSB, we assembled a $\mathrm{Li}|\mathrm{LLZTO}| \mathrm{LiFePO}{ }_{4}$ battery by stacking at $70{ }^{\circ} \mathrm{C}$ the interlayer-covered $\mathrm{LiFePO}_{4}$ cathode, LLZTO, and the interlayer-covered Li metal foil and cool down the battery to RT. The resistance of the SSB was tested with EIS as shown in Figure 3 (c) and (d). The right intercept of the black arc with the real axis allows us to estimate the overall internal resistance of the battery to be $\sim 620 \Omega$. By subtracting the resistance of the LLZTO electrolyte ( $120 \Omega$, see above), the ASR of the electrode|SSE interfaces was calculated to be $\sim 560 \Omega \mathrm{cm}^{2}$. We also compared this result with an SSB without an interlayer. Such an SSB was assembled by first casting and drying the cathode slurry directly on the LLZTO pellet and then attaching a piece of Li metal foil as the anode. To achieve a good contact between Li and LLZTO, the battery was also heated to $150{ }^{\circ} \mathrm{C}$ for 8 hours in vacuum, see details in Figure S3.[35] The interfacial resistance $(\sim 14$ $\mathrm{k} \Omega$ ) of the battery without interlayer is more than 20 times larger than the one with the interlayer. Since the interlayer was applied to both the cathode|SSE and the Li|SSE interfaces, we further investigated the contribution of each interface to the overall resistance. To study the Li|SSE interface, we assembled Li|SSE|Li batteries and carried out EIS measurements, see Figure S4. The resistance of each Li|SSE interface can be estimated by subtracting the Ohmic loss of the LLZTO pellet (120 $\Omega$ ) from the total resistance of the Li|SSE|Li cell $(\sim 3000 \Omega)$, giving an interfacial ASR of $\sim 1600 \Omega \mathrm{cm}^{2}$. We performed identical estimates for the interface that incorporates the interlayer and found that the Li|LLZTO interfacial ASR drops by a factor 16 to $\sim 100 \Omega \mathrm{cm}^{2}$, Figure S5. Also, we computed the resistances of the cathode|SSE interfaces by 
subtracting the contributions of the bulk-SSE and Li|SSE interfaces from the total resistance of the Li|SSE|cathode full cell. As reported in Figure S5, we estimated a drastic decrease of the interfacial ASR (from $\sim 12 \mathrm{k} \Omega \mathrm{cm}^{2}$ to $\sim 400 \Omega \mathrm{cm}^{2}$ ) after incorporation of the interlayer on the cathode side. To study the origin of such a significant drop, we examined the morphology of the cathode|SSE interface. Figure 4 (a) shows the SEM image of the LLZTO|cathode interface without the interlayer. The composite cathode is porous, and the $\mathrm{LiFePO}_{4}$ particles are connected by the conductive carbon and the polyvinylidene fluoride (PVDF) binder. Such a dry mixture has poor Li-ion conductivity because it lacks Li docking sites and transport channels, [36] see Figure 4 (b). In contrast, the interface with the plastic crystal interlayer is significantly more compact as shown in Figure 4 (c). The plastic crystal material not only fills the gap between the SSE pellet and the cathode sheet but also immerses all cathode particles. Since the material is a good ionic conductor, the Li ions not only can travel across the interface but also inside the cathode itself as shown in Figure 4 (d).

The cycling and the rate capability tests of the SSBs are shown in Figure 5. The SSB with the interlayer (Figure 5 (a)) shows a significant improvement over the one without it (Figure 5 (b)). In fact, the SSB without the interlayer can only charge and discharge at an extremely low current density $\left(1 \mu \mathrm{A} \mathrm{cm}^{-2}\right)$. As shown in Figure 5 (b), such SSB shows a charge capacity of $<$ $40 \mathrm{mAh} \mathrm{g}^{-1}$ (active loading is $\sim 1.8 \mathrm{mg} \mathrm{cm}^{-2}$ ), a $1 / 4$ of the theoretical value of $\mathrm{LiFePO}_{4}$. When discharged, only $26 \mathrm{mAh} \mathrm{g}^{-1}$ can be achieved. Since the $\mathrm{LiFePO}_{4}$ particles are only connected via the PVDF and the carbon matrix, the measured loss of capacity can be likely attributed to the poor Li conductivity within the porous cathode and its limited contact with the LLZTO. Also, during charge and discharge, the volume of the $\mathrm{LiFePO}_{4}$ changes by approximately $6 \%$. [37] This volume change may lead to further detachment of the cathode active particles from the LLZTO electrolyte. In turn, the weakened contact likely results in a loss of capacity. When we increased the current density, the measured potential exceeded $5 \mathrm{~V}$, and the test stopped. 
The battery with the doped-SN+FEC interlayer has a significantly better rate performance. With an active material loading of $2 \pm 0.2 \mathrm{mg} \mathrm{cm}^{-2}$, the SSB with such an interlayer achieves a discharge capacity of $140 \mathrm{mAh} \mathrm{g}^{-1}$ at $0.1 \mathrm{C}, 115 \mathrm{mAh} \mathrm{g}^{-1}$ at $0.2 \mathrm{C}, 60 \mathrm{mAh} \mathrm{g}^{-1}$ at $0.5 \mathrm{C}$, and 10 $\mathrm{mAh} \mathrm{g}^{-1}$ at $1 \mathrm{C}$. At higher C-rates, the capacity decreases because of the high ohmic resistance from the LLZTO disk. However, we want to note the low bulk conductivity of the LLZTO electrolyte can be mitigated by making the disc thinner or by substituting the SSE pellet with materials with higher ionic conductivity [8]. The cycling performance of the SSB is demonstrated in Figure 5 (d) and (e). As mentioned previously, the plastic crystal material is unstable against Li metal when there is no FEC additive. This instability can be seen in our $\mathrm{Li}|\mathrm{LLZO}| \mathrm{LiFePO}_{4}$ cell test. As shown in Figure 5 (e), the discharge capacity drops significantly from $\sim 140 \mathrm{mAh} \mathrm{g}^{-1}$ to $\sim 110 \mathrm{mAh} \mathrm{g}^{-1}$ after 50 cycles. When $5 \%$ FEC is added, the capacity remains $\sim 140 \mathrm{mAh} \mathrm{g}^{-1}$ at the $50^{\text {th }}$ cycle.

To investigate the origin of the better cycling stability of interlayer with FEC, we removed LLZTO from the battery and built two model systems using the plastic crystal interlayer material (4 mol.\% LiTFSI doped-SN with and without 5 vol.\% FEC) as the electrolyte, Li metal and $\mathrm{LiFePO}_{4}$ as the cathode material. As explained earlier, the plastic crystal material cannot form a freestanding film. Therefore, we used a glass-fiber separator $(0.8 \mathrm{~mm}$ thick $)$ to accommodate the electrolyte, thereby preventing short-circuiting. The charge and discharge capacities of the Li|plastic crystal| $\mid \mathrm{LiFePO}_{4}$ battery are shown in Figure 6. The battery with the FEC additive shows a stable discharge capacity for 50 cycles with a Coulombic efficiency close to $100 \%$ at $1 \mathrm{C}$. However, the cell without the FEC additive suffers from a significant capacity loss with a Coulombic efficiency as low as $75 \%$. This low efficiency indicates that the electrolyte is likely reacting with the electrodes. Actually, the electrochemical reaction between doped-SN and Li metal at low potentials has been reported in the literature [31] and is also evident from the cyclic voltammetry (CV) tests reported in Figure 2(e). To elucidate the role of FEC in stabilizing the batteries, we carried out X-ray photoelectron spectroscopy (XPS) on 
the cycled negative electrode surfaces, see Figure 7. As shown in Figure 7 (a) and (d), the F 1s spectra indicate that after the inclusion of FEC, a LiF-rich solid electrolyte interphase (SEI) forms on the surface of the Li electrode. This layer is expected to shield the Li surface from further reaction with the solvent molecules. [38-40] The strong interaction of $\mathrm{SN}$ with $\mathrm{Li}$ is also supported by our density functional theory (DFT) calculations: we found a strong binding between the SN molecule and the Li-metal surface with an adsorption energy of $-2.01 \mathrm{eV}$, see Figure 8. In particular, it appears that the $\mathrm{N}$ atom of $\mathrm{SN}$ has a strong affinity with the Li-metal. We further calculated the charge density difference to understand this interaction better. As shown in Figure. 8 (a), the blue and the yellow isosurfaces indicate regions with electron depletion and enrichment. Electron depletion occurs mostly under the Li-metal surface (below the dashed line), indicating a strong electron loss from the metallic Li in favor of SN. Instead, the enrichment occurs majorly in proximity to the SN molecule. Such dipolar electron transfer from $\mathrm{Li}$ to $\mathrm{SN}$ indicates that Li reduces SN. On the other hand, the interaction between the LiFsurface and $\mathrm{SN}$ is significantly weaker $(-0.17 \mathrm{eV})$, and the charge transfer between LiF and $\mathrm{SN}$ is less significant. This weaker interaction suggests that, when $\mathrm{LiF}$ is deposited on the Li surface, the reaction between the negative electrode and $\mathrm{SN}$ is hampered.

To further investigate the reaction between $\mathrm{Li}$ and $\mathrm{SN}$, we melted neat $\mathrm{SN}$ at $70{ }^{\circ} \mathrm{C}$ and dispersed several pieces Li metal into it for 48 hours. As illustrated in Figure S6, the color of SN remarkably changed to yellow. We characterized the product of the reaction by Fouriertransform infrared spectroscopy (FTIR), see Figure S7. In comparison to the neat SN, FTIR of the SN that reacted with $\mathrm{Li}$ has two additional features at $1538 \mathrm{~cm}^{-1}$ and $1623 \mathrm{~cm}^{-1}$, which can be linked to the presence of $\mathrm{C}=\mathrm{O}, \mathrm{C}=\mathrm{C}$, or $\mathrm{C}=\mathrm{N}$ bonds. [41] Since no oxygen is present in our system, these peaks are likely due to $\mathrm{C}=\mathrm{C}$ and $\mathrm{C}=\mathrm{N}$ since nitriles tend to react with $\mathrm{Li}$ form LiCN. $[42,43]$ Therefore, we expect a higher concentration of - $\mathrm{CN}$ groups on the FEC-free Li surface compared with the FEC-containing surface. XPS supports this hypothesis as we found 
that the intensity of the $\mathrm{C} \equiv \mathrm{N}$ features (at $\sim 286.5 \mathrm{eV}$ ) is stronger in the FEC-free sample. This finding is also in agreement with the conclusions from calculations. FEC, which promotes the growth of a LiF-rich layer over the Li metal surface, impedes the reaction of SN with Li, thereby lowering the concentration of $-\mathrm{CN}$-containing residuals. Besides this, another reaction is also possible as suggested by Armand et al. $[42,43]$ who proposed that $\mathrm{SN}$ is polymerized on the Limetal surface. In that case, the reaction product likely consists of polyimines and the $\mathrm{C}=\mathrm{N}$ bond as found in the FTIR spectrum is possibly related to these compounds, see Figure S7. [31, 44] However, we want to note that, due to the ambiguity of the assignment of these peaks in both FTIR and XPS, additional experiments are needed to elucidate the mechanism of interaction between SN and Li.

Ceramic SSEs have been suggested to mitigate the growth of Li dendrites in Li-metal batteries thanks to their rigidity.[45] However, researchers recently found evidence that needle-like dendrites can penetrate through SSEs if the current density is high enough. [46] Dendrites can be even generated in single-crystal SSE at surface defect sites and, subsequently, propagate via a Griffith-like crack extension mechanism. $[47,48]$ The minimum current density that causes the growth of dendrites is called the critical current density (CCD). [49] Sharafi et al. found that Al-doped LLZO grows dendrites even at $60 \mu \mathrm{A} \mathrm{cm}{ }^{-2}$ at RT. [49] Here, we found that the interlayer can significantly increase the CCD of the LLZTO. We verified this by cycling Li symmetric batteries with and without the interlayer at several current densities. As shown in Figure 9 (a-b), the symmetric Li cell without the interlayer displays an unstable voltage at 0.1 $\mathrm{mA} \mathrm{cm}{ }^{-2}$. This is in agreement with the results by Sharafi et al. who obtained a CCD of 0.05 $\mathrm{mA} \mathrm{cm}{ }^{-2}$ for Al-doped LLZO. [49] In contrast, the cell with the doped-SN+FEC interlayer does not short even when subjected to a $0.2 \mathrm{~mA} \mathrm{~cm}^{-2}$ current density. Such an increase is probably due to the improved connection between Li and the SSE. When the contact is weak, the actual current density experienced by the SSE is locally greater than the nominal current density, see 
Figure 9 (d-e). Instead, when a deformable interlayer is added, voids can be filled, and additional Li conduction pathways are provided. This interlayer can, therefore, smooth out the local current density at the Li|SSE interface. Consequently, the measured CCD can increase. We further tested the cyclability of the Li symmetric cells. As shown in Figure 9 (c), the cell with the doped-SN+FEC interlayer could stably run for over 150 hours at $0.2 \mathrm{~mA} \mathrm{~cm}{ }^{-2}$ while the doped-SN interlayer without FEC shows a significant increase in overpotential after 80 hours of cycling, with the battery shorting at $\sim 140$ hours. Such a difference is in agreement with our $\mathrm{CV}$ tests, which show that doped-SN alone is unstable against Li metal. Luckily, this instability can be mitigated by the addition of FEC. [38] After cycling, fluorine-containing species can be detected on the surface of Li thanks to XPS (Figure 7 (a)(d)) and energydispersive X-ray spectroscopy (EDX), see Figure S8.

\section{Conclusions}

Poor solid-solid contact at the interfaces of SSBs lead to significant internal resistances, impeding their practical use at RT. On the cathode side, the lack of Li pathways in the electrode lead to low utilization of the active material. On the anode side, the limited contact area results in hot-spots with high current density which creates dendrites. In this work, we developed a novel strategy to mitigate these interfacial problems by applying a plastic crystal interlayer at the interface between the electrode and the SSE. Such an interlayer has an outstanding Li conductivity (1.01 S cm ${ }^{-1}$ ) and an excellent electrochemically stability (up to $4.5 \mathrm{~V} v s \mathrm{Li} / \mathrm{Li}^{+}$). Thanks to the addition of FEC, an SEI-forming agent, the interlayer also displays a good compatibility with Li metal anode. A interlayer-modified SSB using Li metal as the anode and $\mathrm{LiFePO}_{4}$ as the cathode can stably charge and discharge 50 times at RT with a specific capacity of $140 \mathrm{mAh} \mathrm{g}^{-1}$. Since the interlayer improves the contact between Li and LLZTO interface, the CCD of the LLZTO material is greatly improved. Furthermore interlayer-modified 
Li|LLZTO|Li symmetric cell can stably cycle for over 150 hours at $0.2 \mathrm{~mA} \mathrm{~cm}^{-2}$ without experiencing the growth of dendrites or an increase of the overpotential.

\section{Experimental and Computational Methods}

Preparation of the LLZTO Pellet: The solid electrolyte LLZTO was synthesized via a conventional solid-state method. [34] Stoichiometric amounts of $\mathrm{LiOH} \cdot \mathrm{H}_{2} \mathrm{O}(\geq 99.0 \%$, SigmaAldrich), $\mathrm{La}_{2} \mathrm{O}_{3}\left(99 \%\right.$, Sigma-Aldrich), $\mathrm{Ta}_{2} \mathrm{O}_{5}\left(\geq 99.99 \%\right.$, Ourchem), and $\mathrm{ZrO}_{2}(<100 \mathrm{~nm}$, Sigma-Aldrich) were ball milled in isopropanol at $500 \mathrm{rpm}$ for $90 \mathrm{~min}$. Before ball milling, $\mathrm{La}_{2} \mathrm{O}_{3}$ was dried at $900{ }^{\circ} \mathrm{C}$ in air for $12 \mathrm{~h}$. After that, isopropanol was removed by drying the milled powder at $200{ }^{\circ} \mathrm{C}$ for $12 \mathrm{~h}$. Then, the powder was sintered at $900{ }^{\circ} \mathrm{C}$ in air for $12 \mathrm{~h}$ in a $\mathrm{MgO}$ crucible covered by a lid. After the first sintering cycle, the powder was taken out and an additional 10 wt. $\% \mathrm{LiOH} \cdot \mathrm{H}_{2} \mathrm{O}$ and 0.3 wt. $\% \mathrm{Al}_{2} \mathrm{O}_{3}$ were added before pressing the powder to pellets for the final sintering. The $\mathrm{LiOH} \cdot \mathrm{H}_{2} \mathrm{O}$ was added to compensate for the $\mathrm{Li}$ loss while $\mathrm{Al}_{2} \mathrm{O}_{3}$ helped densify the LLZTO pellets. [50]. The powder was then ground again in isopropanol and uniaxially pressed into small disks at $20 \mathrm{MPa}$ using a cylindrical die (diameter $=16 \mathrm{~mm}$ ) after the removal of the alcohol. Finally, the pellets were sintered at $1140{ }^{\circ} \mathrm{C}$ for 16 $\mathrm{h}$ in $\mathrm{MgO}$ crucibles which were covered with a lid. Mother powder was added on top of the pellet to minimize the Li loss. The prepared disks were then sanded down to the desired thickness.

Preparation of plastic crystal interlayer material: Succinonitrile (SN, $\geq 99.0 \%$, Sigma-Aldrich), fluoroethylene carbonate (FEC, 99\%, Sigma-Aldrich), and lithium bis(trifluoromethanesulfonyl)imide (LiTFSI, $\geq 99.99 \%$, Ourchem) were used without further purification. LiTFSI was dried in vacuum for $24 \mathrm{~h}$ at $140{ }^{\circ} \mathrm{C}$ before use. To obtain the interlayer material, SN was first melted at $70{ }^{\circ} \mathrm{C}$ and 4 mol.\% LiTFSI was added to the melt. After that, different amounts of FEC (either 0 vol.\%, 2.5 vol.\%, 5 vol.\%, 10 vol.\%, or 20 vol.\%,) was 
added to the mixture. The preparation and application of the plastic crystal interlayer material were all done in an Ar-filled glovebox (Mikrouna) with $\mathrm{O}_{2}$ and $\mathrm{H}_{2} \mathrm{O}$ concentrations lower than $0.01 \mathrm{ppm}$.

Materials Characterization: X-ray diffraction (XRD) was performed on PANalytical Empyrean using $\mathrm{Cu} \mathrm{K} \alpha$ radiation $(\lambda=1.5406 \AA)$ in the $2 \theta$ range from $10^{\circ}$ to $90^{\circ}$ at an operation voltage of $40 \mathrm{kV}$ and a current of $40 \mathrm{~mA}$. Scanning electron microscopy (SEM) was carried out on a JEOL-6700F instrument. XPS was performed on electrode surfaces after 30 cycles using a PHI5600 instrument, which is equipped with a monochromatic Al X-ray source.

Electrode preparation: To prepare the cathode sheets, $\mathrm{LiFePO}_{4}$ (Aleees, Taiwan), Super-P carbon black (MTI, TIMICAL SUPER C65), PVDF at an 8:1:1 weight ratio were dispersed in $2 \mathrm{~mL}$ of N-methyl-2-pyrrolidone (NMP, MTI, 99.9\%). The mixture was, first, stirred in a glass bottle using a magnetic stirring bar at $1200 \mathrm{rpm}$ for 8 hours to form a slurry and, then, cast on a carbon coated aluminum foil. Subsequently, the electrodes were dried at $80{ }^{\circ} \mathrm{C}$ in a vacuum oven for $18 \mathrm{~h}$ and cut into disks (diameter $=12 \mathrm{~mm}$ ). The mass loading of each electrode ( $\mathrm{LiFePO}_{4}$ ) was $\sim 2 \mathrm{mg} \mathrm{cm}^{-2}$. The Li metal anodes were prepared by polishing the surface of the Li metal.

Battery assembly: All batteries were assembled using two electrode Swagelok cells (Gelon) with an internal diameter of $13 \mathrm{~mm}$ in the Ar-filled glovebox.

Symmetric Li cell: two pieces of Li metal electrodes were placed on the two stainless steel poles of the Swagelok cell. Then 0 or $5 \mu \mathrm{L}$ of interlayer material was dropped on the Li metal electrodes at $70{ }^{\circ} \mathrm{C}$. After that, the LLZTO pellet was sandwiched between the Li metal electrodes. The battery was then sealed in the Swagelok cell and cooled to RT.

$\mathrm{Li}|\mathrm{LLZTO}| \mathrm{LiFePO}_{4}$ cell: The procedure of assembling the $\mathrm{Li}|\mathrm{LLZTO}| \mathrm{LiFePO}_{4}$ was similar to the one to make the Li symmetric cells. The only difference is that the $\mathrm{LiFePO}_{4}$ cathode was replaced. 
Electrochemical Testing: The charge and discharge of the batteries were performed using a Land CT2001A battery testing system at various current densities. Cyclic voltammetry (CV) tests were conducted between -1 and $5 \mathrm{~V}$ using VSP-300 workstation at a scan rate of $1 \mathrm{mV} \mathrm{s}$

${ }^{1}$. EIS measurement was performed using the same workstation. All electrochemical tests were conducted at RT.

DFT calculations: All DFT calculations were done within the spin-polarized generalized gradient approximation (GGA) of Perdew, Burke, and Ernzerhof (PBE) as implemented in the VASP code. $[51,52]$ Core electrons were treated with the projector augmented wave (PAW) method and the energy cutoff used was $520 \mathrm{eV}$. [53] The energy convergence criterion for the self-consistent loop was set to $10^{-4} \mathrm{eV}$. The $\mathrm{Li}$ and $\mathrm{LiF}$ metal surfaces were constructed using slab models, where at least 5 layers of atoms were included in the slab. The bottom 2 layers were fixed. To avoid interactions between the slabs, a vacuum layer with a height of at least 20 $\AA$ was included. During energy minimization, the atomic positions were allowed to relax using a conjugate gradient algorithm until the energy difference was smaller than $10^{-6} \mathrm{eV}$ and the forces smaller than $10^{-2} \mathrm{eV} / \AA$. The adsorption energy $\left(\mathrm{E}_{\mathrm{ads}}\right)$ was calculated by subtracting the energies of the separated $\mathrm{SN}$ molecule $\left(\mathrm{E}_{\mathrm{SN}}\right)$ and substrate $\left(\mathrm{E}_{\mathrm{Sub}}\right)$ from the energy of the system with adsorbed $\mathrm{SN}\left(\mathrm{E}_{\mathrm{SN}+\text { sub }}\right): \mathrm{E}_{\mathrm{ads}}=\mathrm{E}_{\mathrm{SN}+\text { sub }}-\mathrm{E}_{\mathrm{SN}}-\mathrm{E}_{\mathrm{sub}}$

\section{References}

[1] M. Armand, J.-M. Tarascon, nature, 451 (2008) 652.

[2] B. Scrosati, J. Hassoun, Y.-K. Sun, Energy Environ. Sci. , 4 (2011) 3287-3295.

[3] L. Lu, X. Han, J. Li, J. Hua, M. Ouyang, J. Power Sources 226 (2013) 272-288.

[4] B. Nykvist, M. Nilsson, nature climate change, 5 (2015) 329.

[5] F. Han, Y. Zhu, X. He, Y. Mo, C. Wang, Advanced Energy Materials, 6 (2016) 1501590.

[6] J.C. Bachman, S. Muy, A. Grimaud, H.-H. Chang, N. Pour, S.F. Lux, O. Paschos, F. Maglia, S. Lupart, P. Lamp, Chem. Rev. , 116 (2015) 140-162.

[7] B. Zhang, R. Tan, L. Yang, J. Zheng, K. Zhang, S. Mo, Z. Lin, F. Pan, Energy Storage Materials, (2017).

[8] N. Kamaya, K. Homma, Y. Yamakawa, M. Hirayama, R. Kanno, M. Yonemura, T. Kamiyama, Y. Kato, S. Hama, K. Kawamoto, Nat. Mater. , 10 (2011) 682. 
[9] Y. Mo, S.P. Ong, G. Ceder, Chem. Mater. , 24 (2011) 15-17.

[10] R. Murugan, V. Thangadurai, W. Weppner, Angew. Chem. Int. Ed. , 46 (2007) 77787781.

[11] V. Thangadurai, S. Narayanan, D. Pinzaru, Chem. Soc. Rev. , 43 (2014) 4714-4727.

[12] H. Kawai, J. Kuwano, J. Electrochem. Soc. , 141 (1994) L78-L79.

[13] X. Gao, C.A. Fisher, T. Kimura, Y.H. Ikuhara, H. Moriwake, A. Kuwabara, H. Oki, T.

Tojigamori, R. Huang, Y. Ikuhara, Chem. Mater. , 25 (2013) 1607-1614.

[14] J. Fu, Solid State Ionics 96 (1997) 195-200.

[15] C. Cao, Z.-B. Li, X.-L. Wang, X.-B. Zhao, W.-Q. Han, Frontiers in Energy Research, 2 (2014) 25.

[16] W.D. Richards, L.J. Miara, Y. Wang, J.C. Kim, G. Ceder, Chem. Mater. , 28 (2015) 266273.

[17] T. Liu, Y. Zhang, R. Chen, S.-X. Zhao, Y. Lin, C.-W. Nan, Y. Shen, Electrochem. Commun. , 79 (2017) 1-4.

[18] G. Bucci, T. Swamy, Y.-M. Chiang, W.C. Carter, J. Mater. Chem. A, 5 (2017) 1942219430.

[19] R. Koerver, I. Aygün, T. Leichtweiß, C. Dietrich, W. Zhang, J.O. Binder, P. Hartmann, W.G. Zeier, J.r. Janek, Chem. Mater. , 29 (2017) 5574-5582.

[20] K. Park, B.-C. Yu, J.-W. Jung, Y. Li, W. Zhou, H. Gao, S. Son, J.B. Goodenough, Chem. Mater. , 28 (2016) 8051-8059.

[21] Y. Zhu, X. He, Y. Mo, ACS Appl. Mat. Interfaces 7(2015) 23685-23693.

[22] T. Kato, T. Hamanaka, K. Yamamoto, T. Hirayama, F. Sagane, M. Motoyama, Y.

Iriyama, J. Power Sources 260 (2014) 292-298.

[23] Y. Zhu, X. He, Y. Mo, J. Mater. Chem. A, 4 (2016) 3253-3266.

[24] S. Ohta, S. Komagata, J. Seki, T. Saeki, S. Morishita, T. Asaoka, J. Power Sources 238 (2013) 53-56.

[25] Y. Li, B. Xu, H. Xu, H. Duan, X. Lü, S. Xin, W. Zhou, L. Xue, G. Fu, A. Manthiram, Angew. Chem. Int. Ed. , 56 (2017) 753-756.

[26] W. Luo, Y. Gong, Y. Zhu, Y. Li, Y. Yao, Y. Zhang, K. Fu, G. Pastel, C.F. Lin, Y. Mo, Advanced Materials, 29 (2017) 1606042.

[27] X. Han, Y. Gong, K.K. Fu, X. He, G.T. Hitz, J. Dai, A. Pearse, B. Liu, H. Wang, G.

Rubloff, Nature materials, 16 (2017) 572.

[28] C. Wang, Y. Gong, B. Liu, K. Fu, Y. Yao, E. Hitz, Y. Li, J. Dai, S. Xu, W. Luo, Nano letters, 17 (2016) 565-571.

[29] W. Luo, Y. Gong, Y. Zhu, K.K. Fu, J. Dai, S.D. Lacey, C. Wang, B. Liu, X. Han, Y. Mo, Journal of the American Chemical Society, 138 (2016) 12258-12262.

[30] H. Gao, L. Xue, S. Xin, K. Park, J.B. Goodenough, Angewandte Chemie, 129 (2017) 5633-5637.

[31] P.-J. Alarco, Y. Abu-Lebdeh, A. Abouimrane, M. Armand, Nat. Mater. , 3 (2004) 476.

[32] P. Whitfield, A. Abouimrane, I. Davidson, Solid State Ionics 181 (2010) 740-744.

[33] T.M. Lammens, J. Le Nôtre, M.C. Franssen, E.L. Scott, J.P. Sanders, ChemSusChem, 4 (2011) 785-791.

[34] M. Saccoccio, J. Yu, Z. Lu, S.C. Kwok, J. Wang, K.K. Yeung, M.M. Yuen, F. Ciucci, J. Power Sources 365 (2017) 43-52.

[35] C. Yang, K. Fu, Y. Zhang, E. Hitz, L. Hu, Adv. Mater. , (2017).

[36] F. Du, N. Zhao, Y. Li, C. Chen, Z. Liu, X. Guo, J. Power Sources 300 (2015) 24-28.

[37] D. Wang, X. Wu, Z. Wang, L. Chen, J. Power Sources 140 (2005) 125-128.

[38] X.Q. Zhang, X.B. Cheng, X. Chen, C. Yan, Q. Zhang, Advanced Functional Materials, 27 (2017) 1605989.

[39] W. Xu, J. Wang, F. Ding, X. Chen, E. Nasybulin, Y. Zhang, J.-G. Zhang, Energy Environ. Sci. , 7 (2014) 513-537. 
[40] X.-B. Cheng, R. Zhang, C.-Z. Zhao, Q. Zhang, Chem. Rev. , 117 (2017) 10403-10473. [41] J. Liu, D. Ma, Z. Li, Eur. Polym. J. , 38 (2002) 661-665.

[42] Y. Yamada, K. Furukawa, K. Sodeyama, K. Kikuchi, M. Yaegashi, Y. Tateyama, A. Yamada, J. Am. Chem. Soc., 136 (2014) 5039-5046.

[43] M. Rupich, L. Pitts, K. Abraham, J. Electrochem. Soc. , 129 (1982) 1857-1861.

[44] A. Abouimrane, P. Whitfield, S. Niketic, I. Davidson, J. Power Sources 174 (2007) 883888.

[45] J. Janek, W.G. Zeier, Energy, 500 (2016) 300.

[46] Y. Ren, Y. Shen, Y. Lin, C.-W. Nan, Electrochem. Commun. , 57 (2015) 27-30.

[47] L. Porz, T. Swamy, B.W. Sheldon, D. Rettenwander, T. Frömling, H.L. Thaman, S.

Berendts, R. Uecker, W.C. Carter, Y.M. Chiang, Adv. Energy Mater., 7 (2017).

[48] E. Yi, W. Wang, J. Kieffer, R.M. Laine, J. Mater. Chem. A, 4 (2016) 12947-12954.

[49] A. Sharafi, H.M. Meyer, J. Nanda, J. Wolfenstine, J. Sakamoto, J. Power Sources 302 (2016) 135-139.

[50] Y. Wang, P. Yan, J. Xiao, X. Lu, J.-G. Zhang, V.L. Sprenkle, Solid State Ionics, 294 (2016) 108-115.

[51] J.P. Perdew, K. Burke, M. Ernzerhof, Phys. Rev. Lett. , 77 (1996) 3865.

[52] G. Kresse, J. Hafner, Phys. Rev. B, 47 (1993) 558.

[53] P.E. Blöchl, Phys. Rev. B, 50 (1994) 17953.

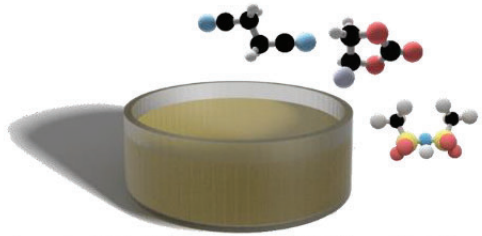

1. mix SN, LiTFSI, and FEC at $70^{\circ} \mathrm{C}$



3. Stack the electrodes and SSE

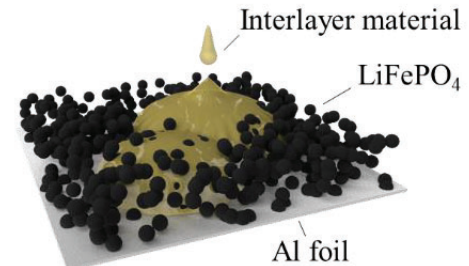

2. drop cast the interlayer at on electrodes

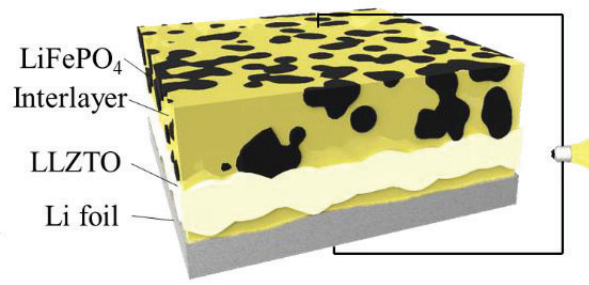

4. solidify the interlayer \& cycle the battery

Figure 1 Illustration of the fabrication and application of the interlayer materials. 
(a)
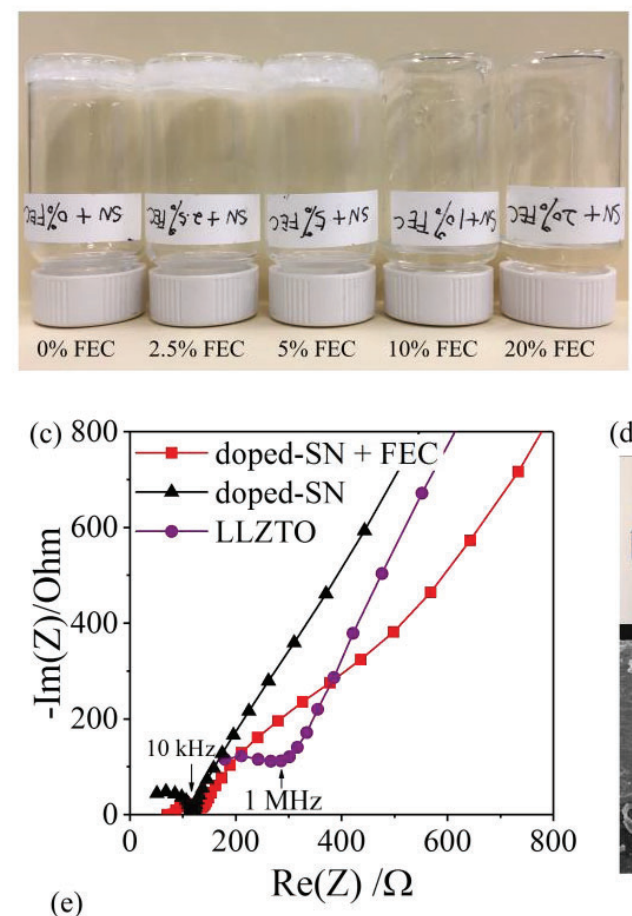

(d)
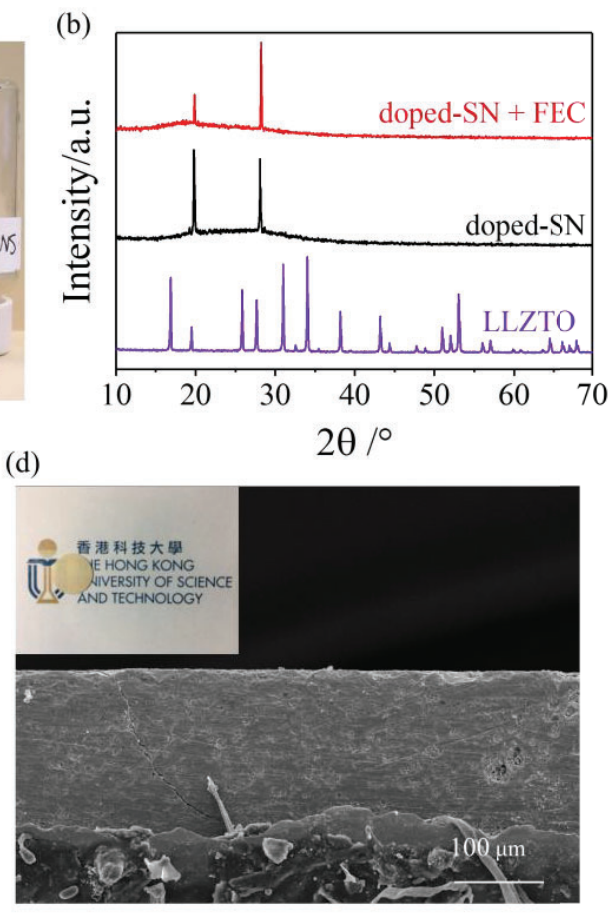

(e)

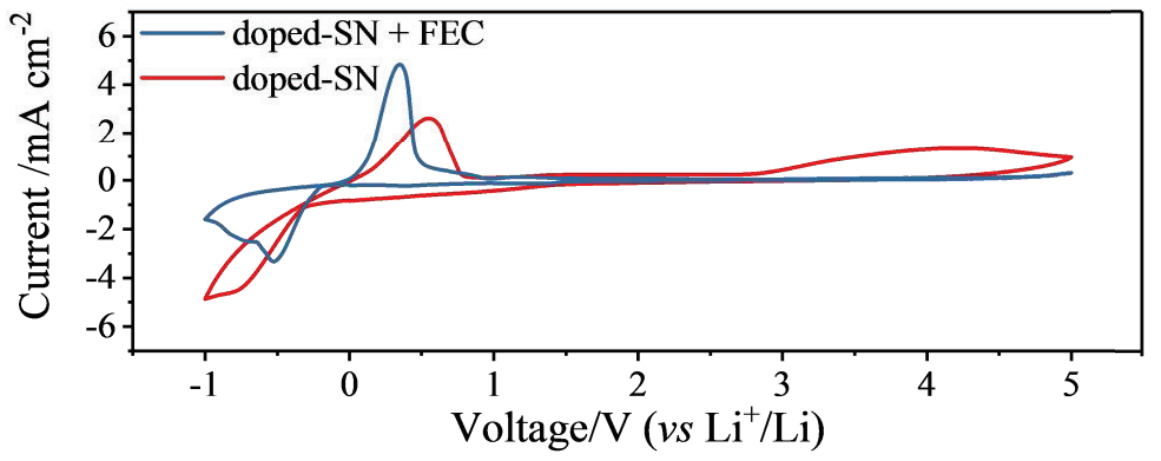

Figure 2 Characterization of the plastic crystal interfacial material and the LLZTO solid electrolyte. (a) Digital images of 4 mol.\% LiTFSI doped-SN with different amounts of FEC at RT. (b) XRD pattern of LLZTO and of 4 mol.\% LiTFSI-doped SN with (denoted as doped-SN) and without an additional 5 vol.\% FEC (denoted as doped-SN+FEC). (c) The EIS of doped-SN, doped-SN+FEC, and the LLZTO pellet using blocking electrodes. (d) SEM of the cross-section of the LLZTO pellet. (e) CV of doped-SN, doped-SN + FEC with Li metal as a counter electrode. 

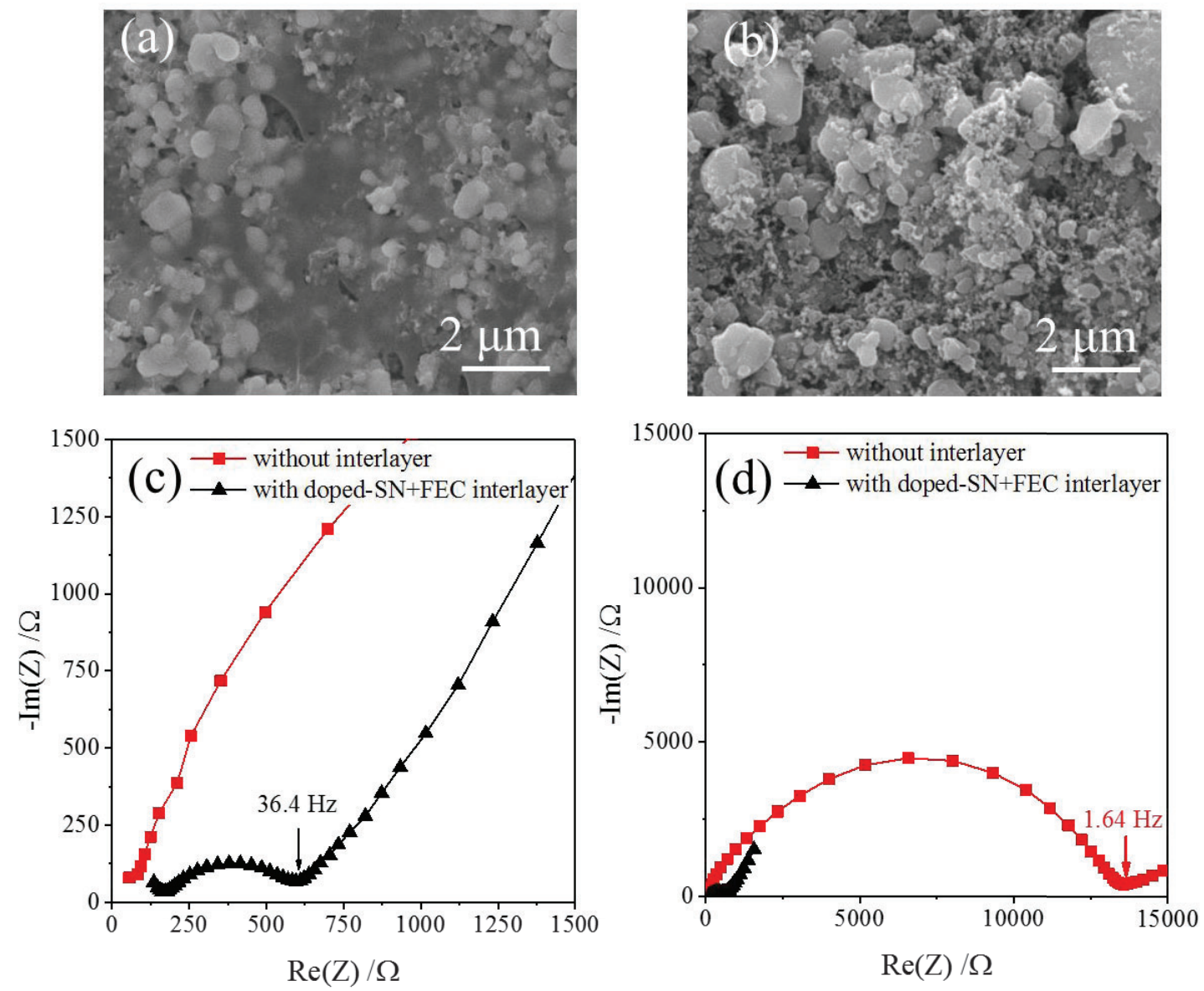

Figure $3 \mathrm{SEM}$ of the $\mathrm{LiFePO}_{4}$ cathode (a) with and (b) without the doped-SN+FEC interlayer material on top; (c) EIS of the $\mathrm{Li}|\mathrm{LLZTO}| \mathrm{LiFePO}_{4} \mathrm{SSBs}_{\text {with }}$ and without the interlayer. In (d), the range was enlarged. 

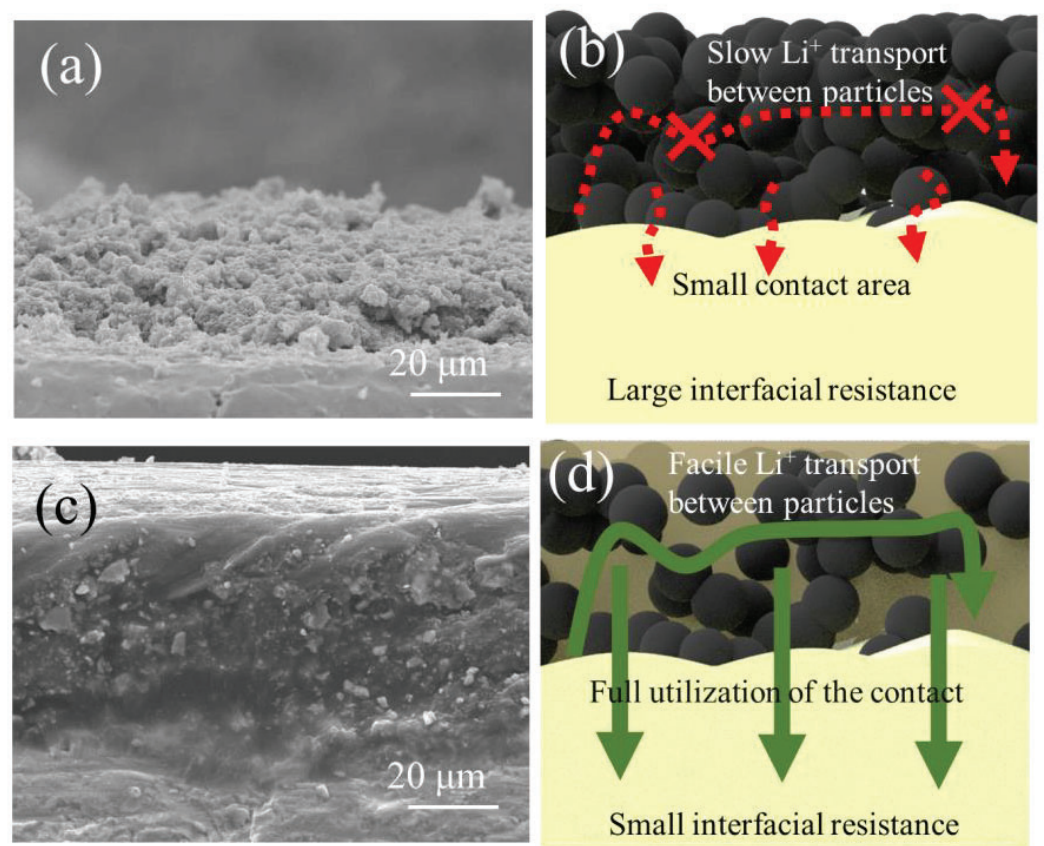

Figure 4 SEM of the cathode|LLZTO interface (a) without and (c) with interlayer. Schematic illustration of the $\mathrm{Li}^{+}$fluxes at the cathode|LLZTO interface (b) without and (d) with interlayer 

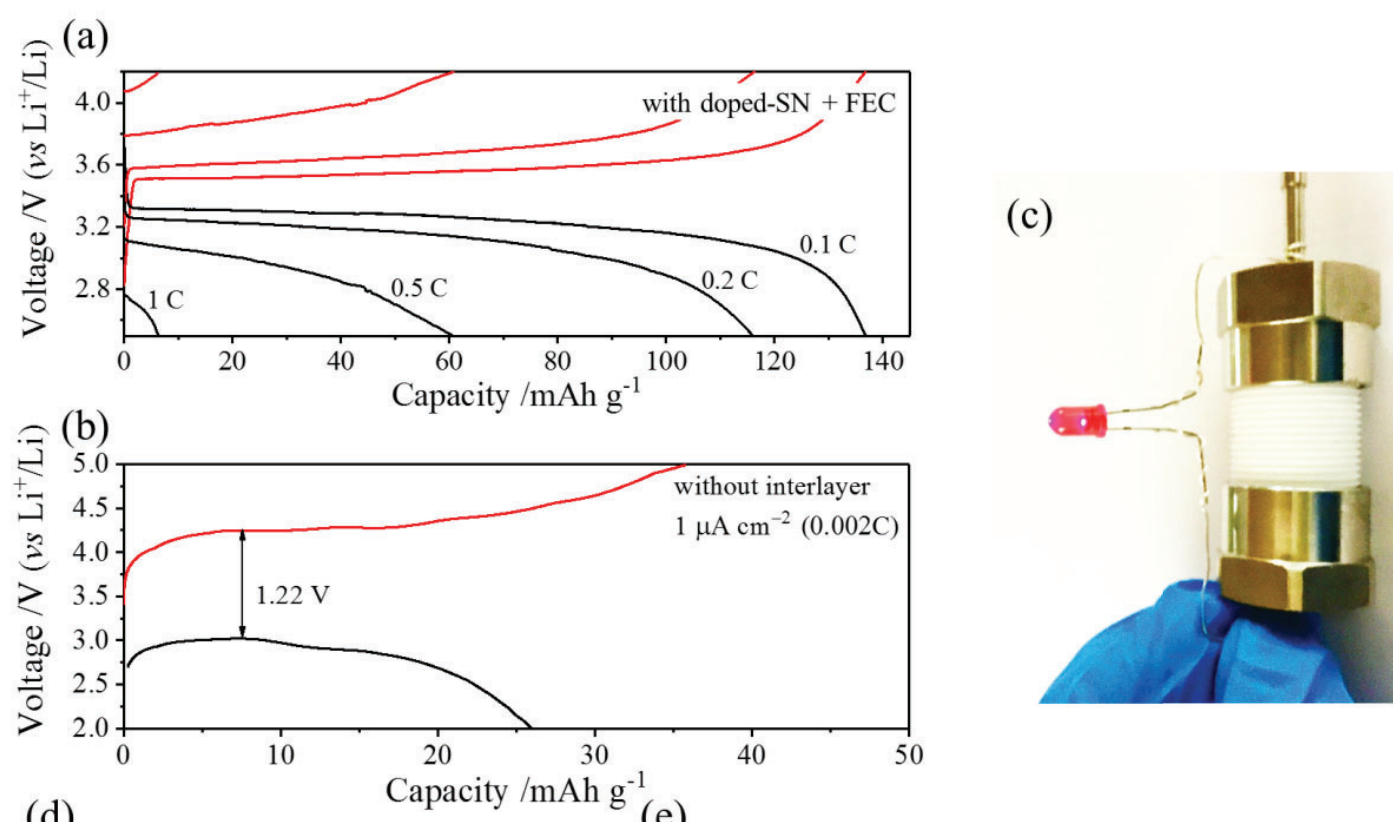

(d)

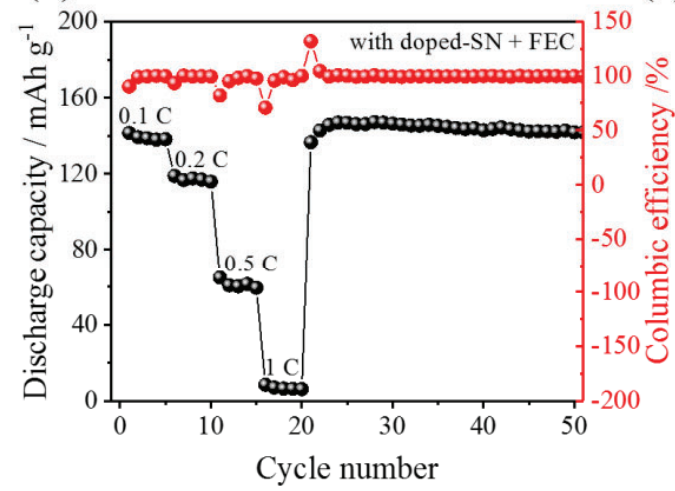

(e)

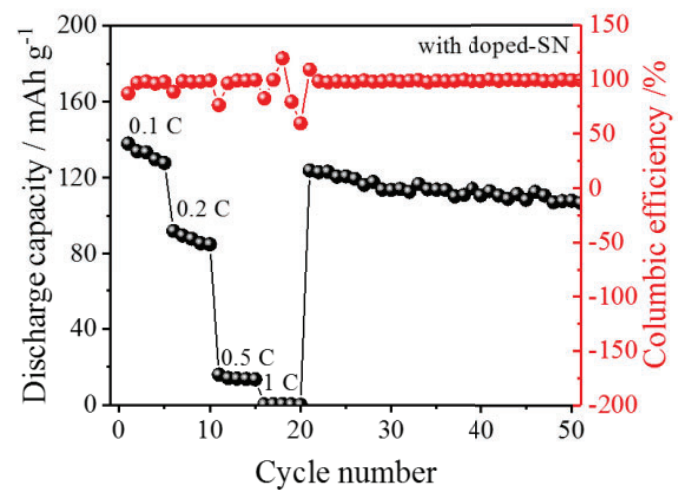

Figure 5 Cycling the $\mathrm{Li}|\mathrm{LLZTO}| \mathrm{LiFePO}_{4}$ batteries. Charge and discharge curve of

$\mathrm{Li}|\mathrm{LLZTO}| \mathrm{LiFePO}_{4}$ batteries (a) with doped-SN+FEC interlayer and (b) without any interlayer.

(c) Lighting an LED with the SSB. Cycling and rate performance of $\mathrm{Li}|\mathrm{LLZTO}| \mathrm{LiFePO}_{4}$ battery with (d) doped-SN+FEC and (e) doped-SN as interlayers.
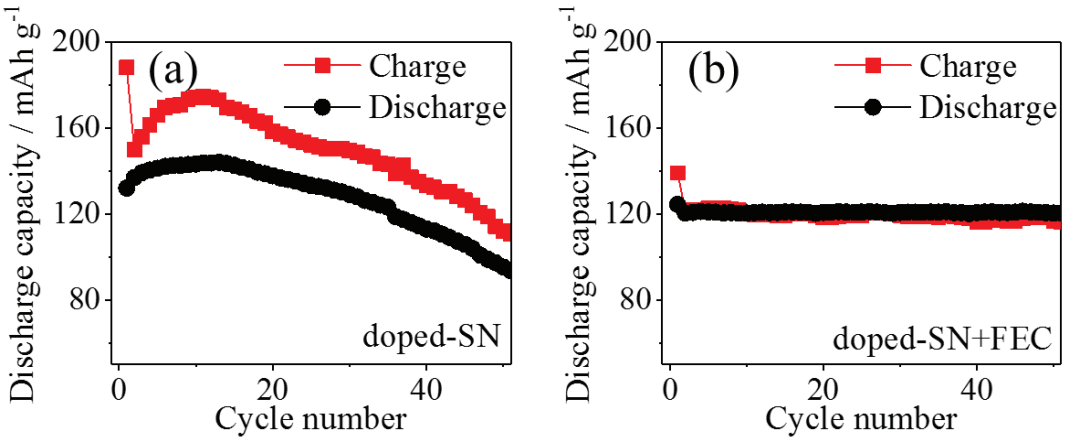
Figure 6 Specific capacity of Li|plastic crystal|LiFePO 4 cell with (a) LiTFSI doped-SN and (b) LiTFSI doped-SN+5 vol. \% FEC.
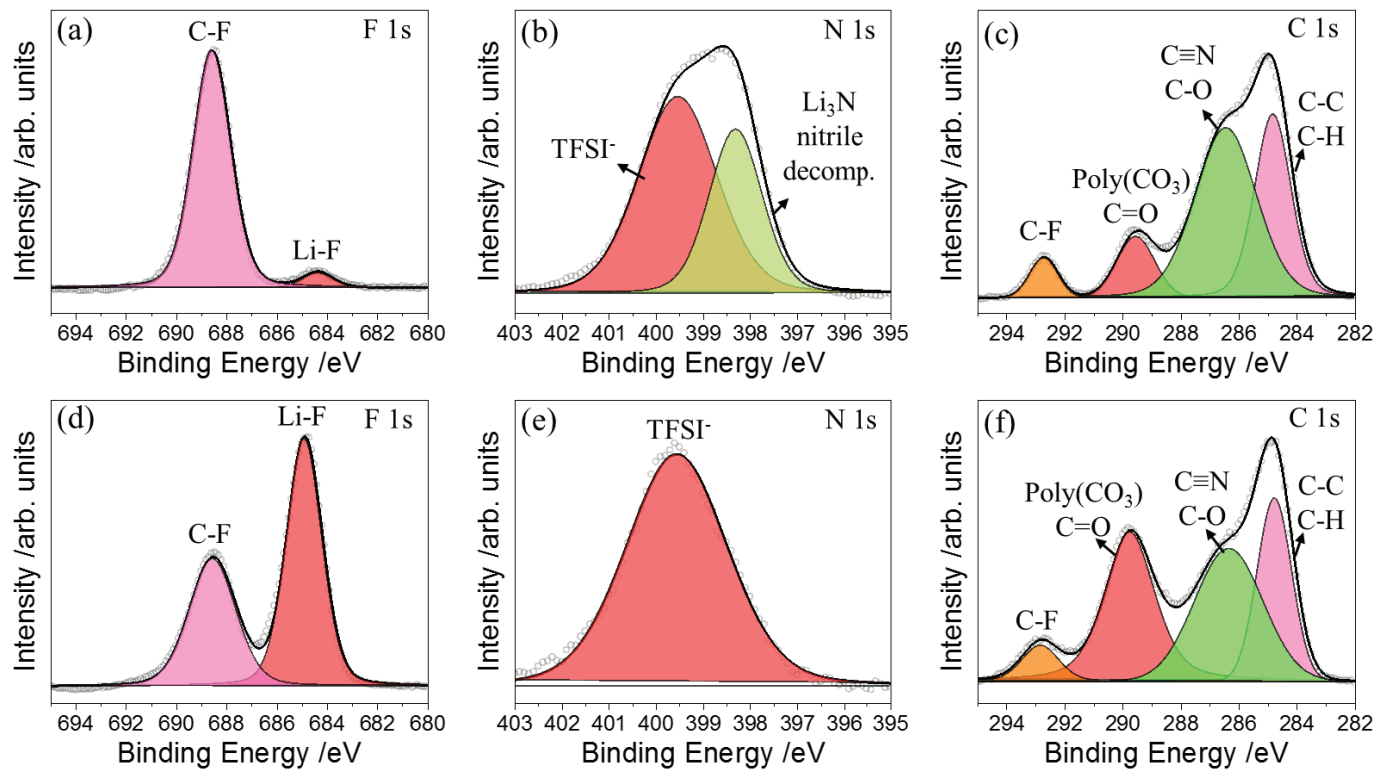

Figure 7 XPS of the Li metal surfaces after cycling in doped-SN (a-c) and doped-SN+FEC (d-

f).

(a) $\mathrm{E}_{\mathrm{ads}}=-2.01 \mathrm{eV}$

(b)

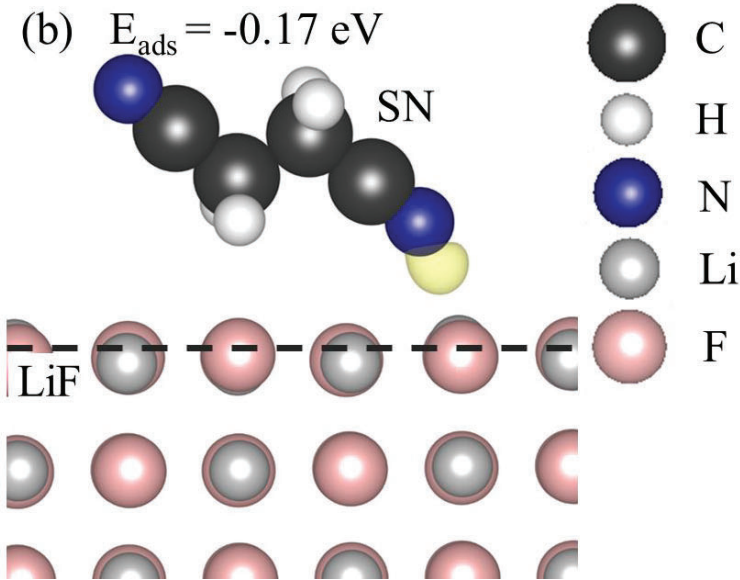

- $\mathrm{C}$

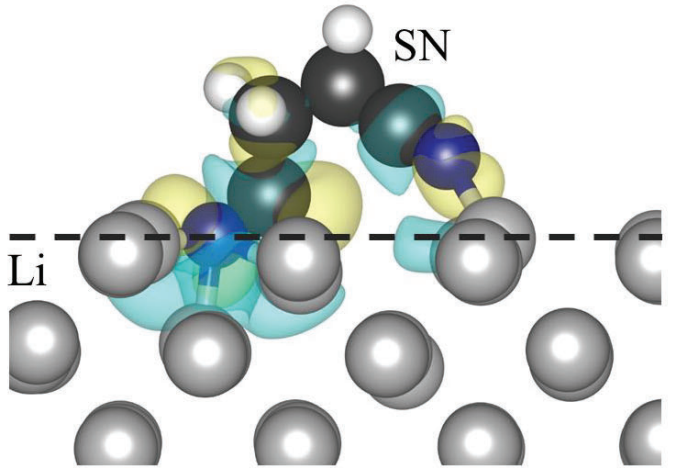

Figure 8 Adsorption of an SN molecule on (a) Li-metal and (b) LiF surfaces. The charge

difference is calculated by subtracting the electron density of the SN molecule and the substrate from the density of the system with SN adsorbed. The yellow and blue isosurfaces show the charge difference at $\pm 0.002|\mathrm{e}| / \AA^{3}$, representing enrichment and depletion of electrons after the adsorption of SN molecule, respectively. 

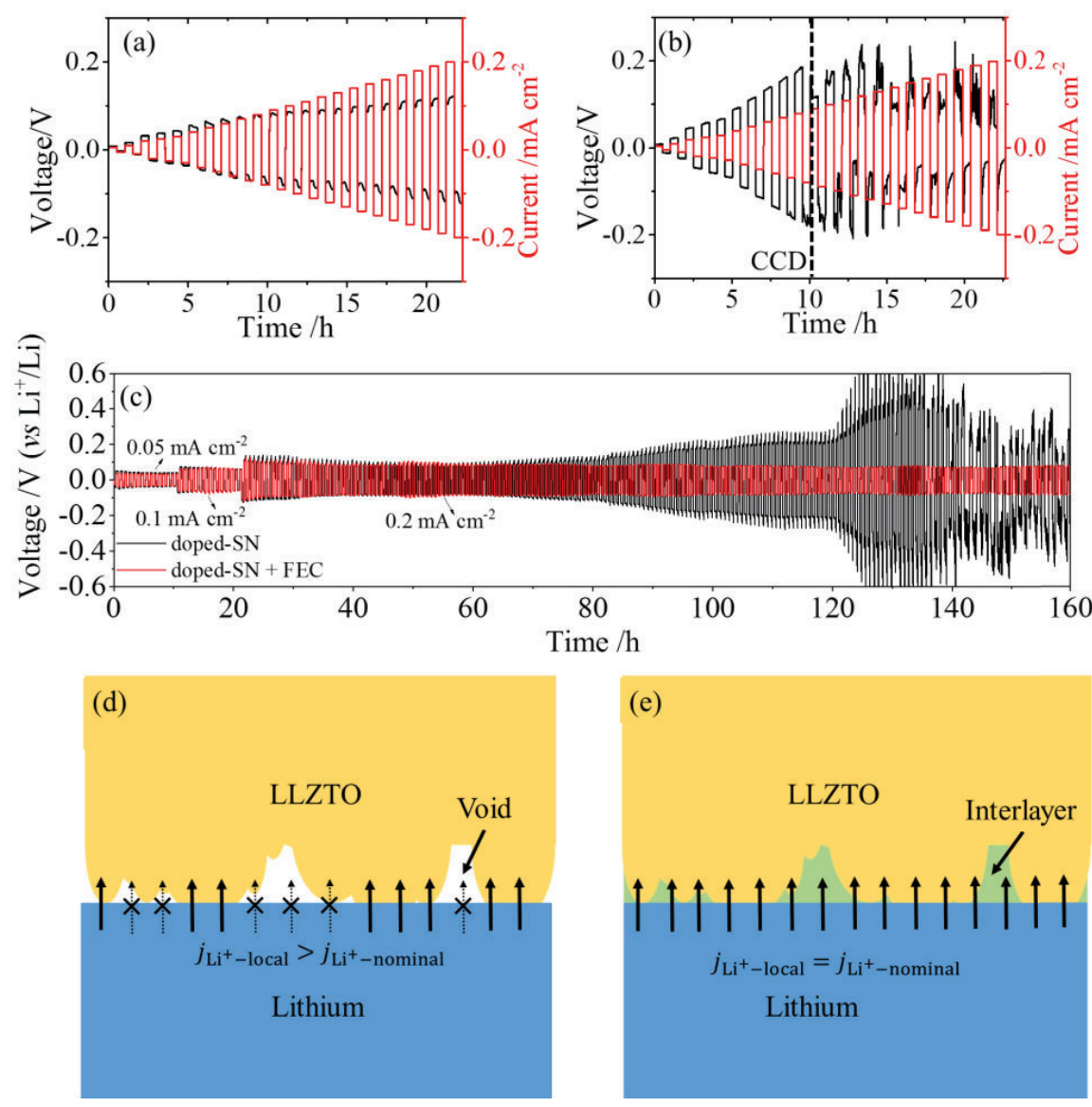

Figure 9 CCD test conducted on a Li|LLZTO|Li cell (a) with the doped-SN+FEC interlayer and (b) without the interlayer. (c) Li|LLZTO|Li symmetric cell cycling tests with doped-SN and doped-SN+FEC interlayers. Schematic illustration of charge transfer on Li|LLZTO interfaces (d) with and (e) without the interlayer. 